\title{
Detection of spin polarization in quantum point contacts
}

\author{
Leonid P Rokhinson ${ }^{1}$, Loren N Pfeiffer ${ }^{2}$ and Ken W West ${ }^{2}$ \\ ${ }^{1}$ Department of Physics and Birck Nanotechnology Center, Purdue University, \\ West Lafayette, IN 47907, USA \\ ${ }^{2}$ Bell Laboratories, Lucent Technologies, Murray Hill, NJ 07974, USA \\ E-mail: leonid@physics.purdue.edu
}

Received 10 October 2007

Published 1 April 2008

Online at stacks.iop.org/JPhysCM/20/164212

\begin{abstract}
We use spatial spin separation by a magnetic focusing technique to probe the polarization of quantum point contacts. The point contacts are fabricated from p-type GaAs/AlGaAs heterostructures. A finite polarization is measured in the low density regime, when the conductance of a point contact is tuned to $<2 e^{2} / h$. Polarization is stronger in samples with a well defined ' 0.7 structure'.
\end{abstract}

(Some figures in this article are in colour only in the electronic version)

\section{Introduction}

Mesoscopic systems exhibit a range of non-trivial spinrelated phenomena in the low density regime, where interparticle Coulomb interactions become comparable to their kinetic energy. In zero-dimensional systems spontaneous polarization of a few electron quantum dots leads to a spin blockade [1-3], a remarkable effect where mismatch of a single spin blocks macroscopic current flow. In two-dimensional hole gases there is experimental evidence of a finite spin polarization even in the absence of a magnetic field [4]. In one-dimensional systems-quantum wires and quantum point contacts-a puzzling so-called ' 0.7 structure' has been observed below the first quantization plateau [5]. Experiments suggest [5-8] that an extra plateau in the conductance versus gate voltage characteristic at $0.7 \times 2 e^{2} / h$ is spin related. However, the origin of the phenomenon is not yet understood and is highly debated.

Quantization of ballistic conductance $G$ in integer multiples of $g_{0}=2 e^{2} / h$ is a fundamental property of $1 \mathrm{D}$ systems $[9,10]$, which originates from the exact cancelation of velocity and the 1D density of state. Each energy level below the Fermi energy inside a 1D channel contributes $0.5 g_{0}$ to the total conductance. An extra factor of two accounts for the spin degeneracy. Strong magnetic fields can lift the degeneracy; in this case quantization in multiples of $0.5 g_{0}$ is observed. This single-particle result is robust even in the presence of electronelectron interactions because they preserve the center-of-mass velocity of the scattered electrons. Thus, observation of a quantized plateau at $0.7 g_{0}$ in the absence of a magnetic field in $\mathrm{n}-\mathrm{GaAs}$ [5], $\mathrm{n}-\mathrm{GaN}$ [11] and p-GaAs QPCs pose a serious challenge to our understanding of 1D conductors.

Phenomenologically, the observed structure can be explained if one assumes the existence of static spin polarization at zero magnetic field and confinement-dependent spin splitting of the spin subbands [12]. The well-known Lieb-Mattis theorem forbids polarization in 1D systems [13], albeit that it excludes materials with spin- or velocitydependent forces, such as spin-orbit (SO) interactions. Some theories suggest possible deviation from this theorem in a realistic channel with finite width [14-16]. Recently, it has been pointed out that temperature and bias dependence of differential conductance around the $0.7 g_{0}$ plateau are similar to the Kondo phenomena, thus suggesting dynamic spin polarization [7, 17-19]. Alternative theories assume no polarization and attribute the phenomena to electronphonon interactions [20] or formation of a Wigner crystal [21]. Thus, experimental measurements of spin polarization should provide the most direct method to distinguish between different possibilities for the microscopic origin of the ' 0.7 structure'.

\section{Spin separation and detection by magnetic focusing}

Magnetic focusing of ballistic carriers has been used to investigate properties of Fermi surfaces in metals [22-24] and semiconductors [25]. Using this method, the existence of 

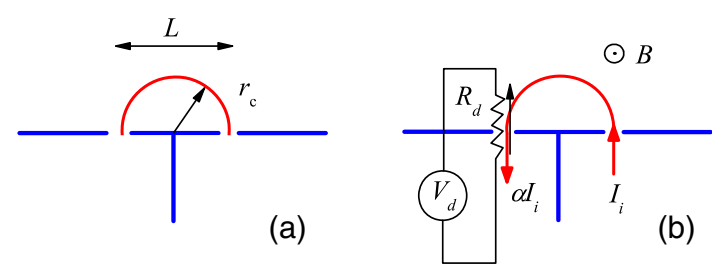

Figure 1. (a) Schematic of magnetic focusing. (b) Origin of the voltage across the detector point contact.

new quasiparticles in fractional quantum Hall effect regimecomposite fermions-has been verified [26]. Later the method has been used to spatially separate carriers with opposite spins [27]. The method resembles mass spectrometry and requires ballistic transport of carriers between two collinear injector and detector contacts, figure 1(a). The carriers, injected from an injector contact, are bent in the direction of the collector by an external magnetic field, when contact separation equals integer multiple of the cyclotron diameter $L=\mathrm{i} 2 r_{\mathrm{c}}$ they are focused into the detector contact resulting in a measurable voltage.

In the case of parabolic dispersion and in the absence of SO interaction $r_{\mathrm{c}}=\frac{\hbar k_{\mathrm{f}}}{e B}$, and magnetic focusing conditions will be satisfied every time $B=\mathrm{i} \frac{2 \hbar k_{\mathrm{f}}}{e L}$, where $\hbar$ is the reduced Plank's constant, $k_{\mathrm{f}}=\sqrt{2 m \epsilon_{\mathrm{f}}} / \hbar, \epsilon_{\mathrm{f}}$ is the Fermi energy, and $m$ and $e$ are the carriers' effective mass and electrical charge.

Spin-orbit interactions drastically modifies the dispersion relation, figure 2. For simplicity, lets assume that charge carriers in GaAs quantum well are characterized by isotropic kinetic energy and the Dresselhaus SO interaction, so that the Hamiltonian can be written as [28] $H=\frac{1}{2 m}\left(p_{x}+\gamma \sigma_{x}\right)^{2}+$ $\frac{1}{2 m}\left(p_{y}-\gamma \sigma_{y}\right)^{2}$, where $\vec{p}$ is the electron momentum, $\sigma_{i}$ are the Pauli matrices $(i=x, y)$, and $\gamma$ is the SO parameter. Similar results can be obtained starting with Rashba Hamiltonian. We will also neglect anisotropy of the effective mass that do not change the qualitative picture. In the semiclassical description, appropriate for the range of magnetic fields $B_{\perp}$ used for the focusing, the motion is described by simple equations

$$
\begin{gathered}
\frac{\mathrm{d} \vec{p}}{\mathrm{~d} t}=e \vec{v} \times \vec{B} \quad \vec{v}=\frac{\mathrm{d} \vec{r}}{\mathrm{~d} t}=\frac{\partial \epsilon_{ \pm}(\vec{p})}{\partial \vec{p}} \\
\epsilon_{ \pm}=\frac{1}{2 m}(p \pm \gamma)^{2}+\frac{\gamma^{2}}{2 m},
\end{gathered}
$$

where $\vec{r}, \vec{v}$ and $\epsilon_{ \pm}$are the charge carrier coordinate, velocity and energy for the two spin projections. This description implies that carrier wavelength is smaller than the cyclotron radius, and that jumps between orbits with different spin projections are absent, i.e. $\epsilon_{\mathrm{f}} \gg \gamma p / m \gg \hbar \omega_{\mathrm{c}}$. Equations (1) show that the charge carrier with energy $\epsilon_{ \pm}=\epsilon_{\mathrm{f}}$ is characterized by the spin-dependent momentum $p^{ \pm}$, cyclotron radius $r_{\mathrm{c}}^{ \pm}$and cyclotron frequency $\omega_{\mathrm{c}}^{ \pm}$. The solution to these equations is

$$
\begin{gathered}
p^{ \pm}=\sqrt{2 m\left(\epsilon_{\mathrm{f}}-\gamma^{2} / m\right)} \pm \gamma \\
r_{\mathrm{c}}^{ \pm}=\sqrt{2 m\left(\epsilon_{\mathrm{f}}-\gamma^{2} / m\right)} / m \omega_{\mathrm{c}}^{ \pm} \\
\omega_{\mathrm{c}}^{ \pm}=\frac{e B_{\perp}}{m}\left(1 \pm \gamma / p_{ \pm}\right) .
\end{gathered}
$$
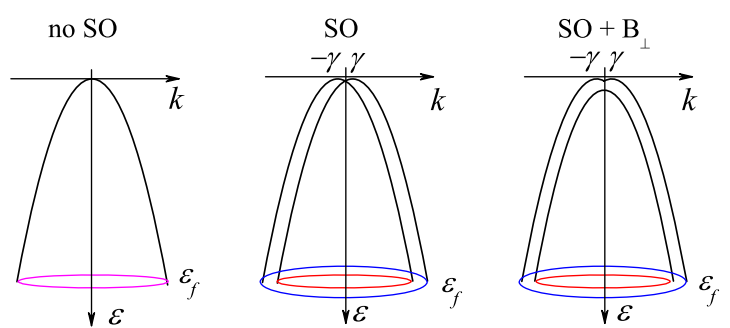

Figure 2. Schematic of energy dispersion with and without spin-orbit (SO) interactions.

Using the semiclassical limit of the quantum description, one obtains the identical results $[29,30]$. In the above equations we ignored Zeeman contribution to the carriers energy which should be added under the radical and leads to the lifting of the degeneracy at $k=0$. The omission is justified for $g_{\perp}^{*} \mu_{\mathrm{B}} B_{\perp} \ll \epsilon_{\mathrm{f}}$, where $g_{\perp}^{*}$ is a normal component of Landé $g$-factor and $\mu_{\mathrm{B}}$ is the Bohr's magneton.

The cyclotron motion in the presence of spin-orbit interactions is spin dependent and the focusing condition for the first focusing peak will be satisfied for the two spin orientations at different fields

$$
B_{\perp}^{ \pm}=2\left(p_{\mathrm{f}} \mp \gamma\right) / e L
$$

Thus, if broadening of the focusing peaks is less than their separation $B_{\perp}^{+}-B_{\perp}^{-}=4 \gamma / e L$ each spin component of the injector current can be measured separately. In order to quantify the amount of polarization one can follow the analysis of Potok et al [31]. The $\alpha I_{i}$ portion of the total injector current $I_{i}=I_{i}^{+}+I_{i}^{-}$is ballistically injected into the detector contact when appropriate focusing conditions are met, see schematic in figure 1(b). The excess charge flows back through the detector point contact and generates voltage $V_{\mathrm{d}}=\alpha I_{i} R_{\mathrm{d}}\left(1+P_{\mathrm{i}} P_{\mathrm{d}}\right)$ across the detector. Here, $R_{\mathrm{d}}$ is the resistance of the detector point contact and $P_{\mathrm{i}}$ and $P_{\mathrm{d}}$ are polarizations of the detector and the injector. In the particular case of spin detection by magnetic focusing the detector is tuned to accept both polarizations equally, equivalent to $P_{\mathrm{d}}=+1$ for $B_{\perp}=B_{\perp}^{+}$and $P_{\mathrm{d}}=-1$ for $B_{\perp}=B_{\perp}^{-}$. Thus, polarization of the injector point contact can be determined as

$$
P_{\mathrm{i}}^{(1)}=\left(V_{\mathrm{d}}^{+}-V_{\mathrm{d}}^{-}\right) /\left(V_{\mathrm{d}}^{+}+V_{\mathrm{d}}^{-}\right),
$$

where $V_{\mathrm{d}}^{ \pm}$are the detector voltages for the two focusing conditions $B_{\perp}=B_{\perp}^{ \pm}$. Alternatively, injector polarization can be deduced from the analysis of each spin component separately

$$
P_{\mathrm{i}}^{(2)}=\left|V_{\mathrm{d}}^{ \pm} / V_{d 0}^{ \pm}-1\right|,
$$

where $V_{d 0}^{ \pm}$are detector voltages for the case of unpolarized injector.

\section{Measurements of QPC polarization}

\subsection{Sample preparation}

In order to resolve SO-split focusing peaks narrow injector and detector are required. It has been noticed that atomic 


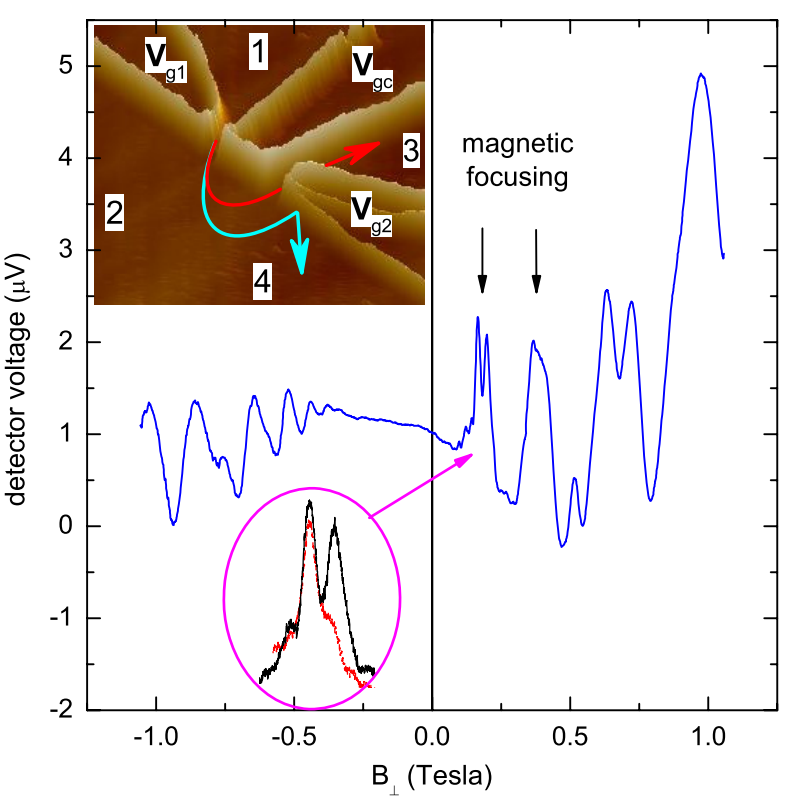

Figure 3. Magnetoresistance and layout of focusing devices. Voltage across the detector (contacts 3 and 4 ) is measured as a function of magnetic field perpendicular to the surface of the sample $\left(B_{\perp}\right)$. Current of $1 \mathrm{nA}$ is flowing through the injector (contacts 1 and 2). Positions of the magnetic focusing peaks are marked with arrows. Top inset: AFM micrograph of sample A $(5 \mu \mathrm{m} \times 5 \mu \mathrm{m})$. Light lines are the oxide which separates different regions of 2D hole gas. Red and blue semicircles show schematically the trajectories for two spin orientations. Bottom inset: first focusing peak measured in the presence of in-plane field $B_{\|}=3.5 \mathrm{~T}$ with injector conductance $G_{i}=g_{0}$ (black solid line) and $G_{i}=1 / 2 g_{0}($ red dashed line $)$

force microscopy local anodic oxidation technique (AFM LAO) [32, 33] provides sharper potential compared with electrostatic top gating. Our devices are fabricated from a twodimensional hole gas (2DHG) using an AFM LAO. Oxide lines separate the 2DHG underneath by forming $\sim 200 \mathrm{mV}$ potential barriers. Several specially designed heterostructures are grown by MBE on [113]A GaAs [34]. Despite very close proximity to the surface ( $350 \AA$ ), the 2 DHG has exceptionally high mobility $\sim 0.5 \times 10^{6} \mathrm{~V} \mathrm{~s} \mathrm{~cm}^{-2}$. Devices are fabricated from two wafers with hole densities $p=1.47 \times 10^{11} \mathrm{~cm}^{-2}$ (wafer A) and $p=0.9 \times 10^{11} \mathrm{~cm}^{-2}$ (wafer B). For quantitative analysis we use data collected during a single cooldown for each device, the qualitative features are reproducible upon several thermal cyclings.

Devices consist of two QPCs separated by a central gate, see inset in figure 3. The potential inside the point contacts can be controlled separately by the two side gates $V_{\mathrm{g} 1}$ and $V_{\mathrm{g} 2}$, or by the central gate $V_{\mathrm{gc}}$. Magnetoresistance $R\left(B_{\perp}\right)$ is measured by applying small current through the injector QPC (contacts 1 and 2) while monitoring voltage across the detector QPC (contacts 3 and 4), see inset in figure 3. At $B_{\perp}<0$ cyclotron motion forces carriers away from the detector. Than, only $2 \mathrm{DHG}$ contributes to $R$, which has almost no dependence on $B_{\perp}$ at low fields and shows Shubnikovde Haas oscillations at $\left|B_{\perp}\right|>0.3 \mathrm{~T}$. For $B_{\perp}>0$ several peaks due to magnetic focusing are observed. Peaks separation $\Delta B \approx 0.18 \mathrm{~T}$ is consistent with the expected value for the

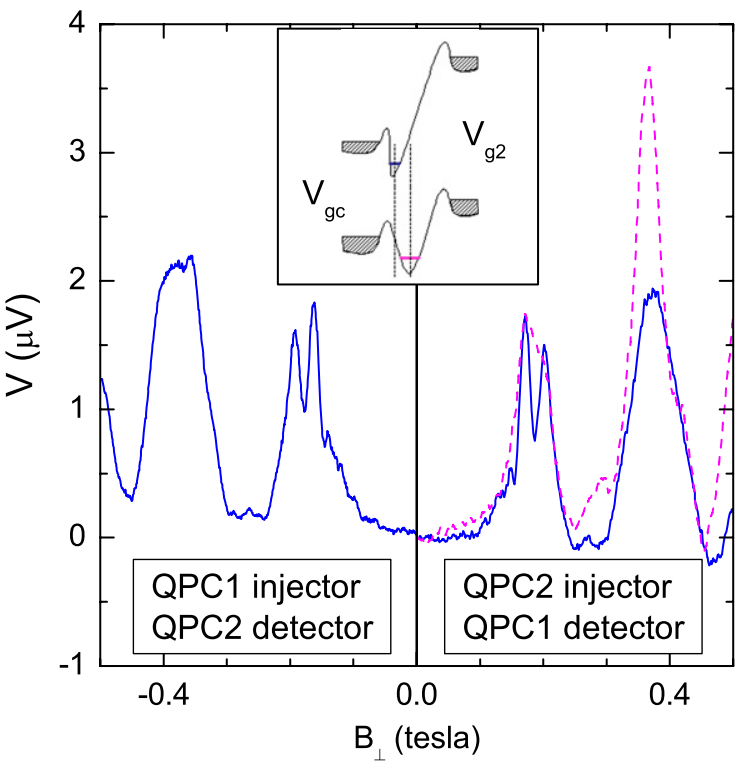

Figure 4. Magnetic focusing is symmetric upon field inversion and simultaneous exchange of injector and detector contacts. Application of differential voltage across the injector and detector QPCs makes potential sharper, as shown schematically in the inset, and focusing peaks narrower: solid blue (dashed magenta) lines are for $V_{\mathrm{g} 2}-V_{\mathrm{gc}}=0.46(0.37) \mathrm{V}$.

lithographical distance between the injector and detector QPCs $L=0.8 \mu \mathrm{m}$. The data is symmetric upon exchange of the injector and detector and simultaneous reversal of the magnetic field as shown in figure 4.

The first focusing peak is a doublet consisting of two peaks separated by $36 \mathrm{mT}$. These peaks are the sharpest when both QPCs are gated to pass exactly one spin-degenerate mode (within the $G=g_{0}$ conductance plateau in the QPC characteristic). We also applied differential voltage between the central and the side gates. Asymmetric biasing of QPCs provides sharper confining potential (see schematic in figure 4) and reduces the distance between the two potential minima by $\Delta L \sim 0.07 \mu \mathrm{m}$. At zero in-plane magnetic field peaks in the doublet have approximately the same height. If in addition to the small orbital field we apply $B_{\|}=3.5 \mathrm{~T}$ inplane magnetic field we can Zeeman-split energy levels and fully polarize the injected current by tuning conductance of the injector QPC to $G_{i}=1 / 2 g_{0}$ plateau. In this case only one peak remains visible in the doublet, see red dashed line in the inset in figure 3. For comparison, both peaks in the doublet are present (the black solid line) for $G_{i}=g_{0}$, where both polarizations are equally present in the injected current. Thus, the two peaks within the first focusing peak correspond to the focusing conditions for the two orthogonal spin states in the $2 \mathrm{DHG}$ and are adiabatically related to the pure spin states inside the point contacts [27]. Thus equations (4) and (5) can be used to analyze the injector polarization.

\section{2. '0.7 structure' in p-GaAs $Q P C$}

Conductance of point contact QPC1 is plotted in figure 5 as a function of the gate voltage $V_{\mathrm{g} 1}$. At zero field (leftmost curve) 


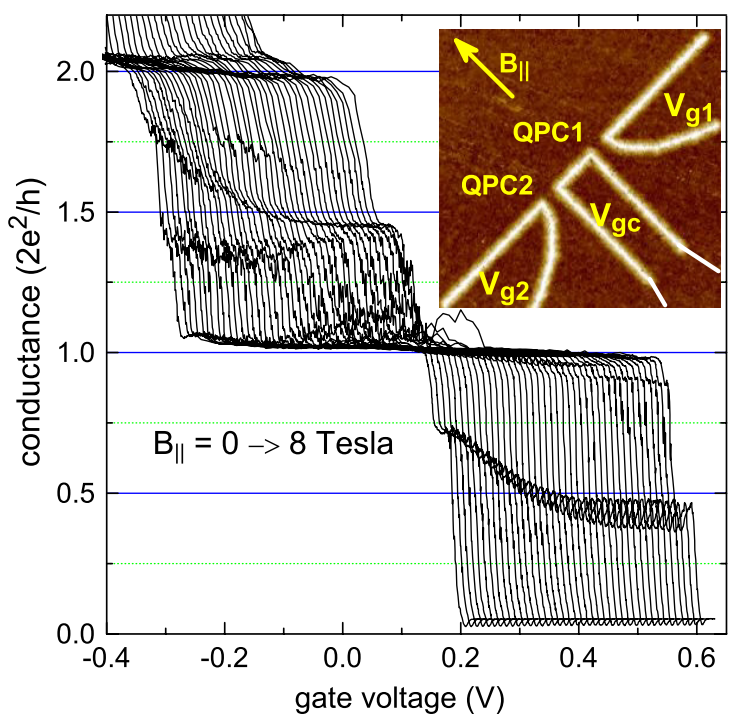

Figure 5. Conductance of the injector QPC1 $G_{i}$ is plotted as a function of the gate voltage $V_{\mathrm{g} 1}$ for in-plane magnetic fields $0<B_{\|}<8 \mathrm{~T}$ at temperature $T=50 \mathrm{mK}$. Curves offset proportionally to $B_{\|}$; the leftmost is $B_{\|}=0$. Inset: AFM micrograph of a sample $(3.3 \mu \mathrm{m} \times 3.3 \mu \mathrm{m})$. The direction of $B_{\|}$is indicated by the arrow.

plateaus with conductance quantized at $g_{0}$ and $2 g_{0}$ are clearly observed. In addition, an extra plateau can be seen at $G \sim 0.7 g_{0}$ and, less developed, at $G \sim 1.7 g_{0}$. When an in-plane magnetic field $B_{\|}$is applied, the $0.7 g_{0}$ and $1.7 g_{0}$ plateaus gradually shift toward $0.5 g_{0}$ and $1.5 g_{0}$, saturating for $B_{\|}>4 \mathrm{~T}$. This gradual decrease is different from the abrupt appearance of half-integer plateaus for higher energy levels. In that case plateaus become more prominent as Zeeman splitting increases, but conductance values of the plateaus do not change with $B_{\|}$, consistent with the single-particle picture.

Another signature of ' 0.7 structure' is the anomalous nonlinear differential conductance $g=\mathrm{d} I / \mathrm{d} V$. A distinct peak in $g$ versus dc bias $V_{\text {bias }}$ has been reported in electron QPCs [7]. Nonlinear conductance in our hole device is analyzed in figure 6 . Indeed, there is a well developed zerobias peak at the lowest $T=25 \mathrm{mK}$ and $B_{\|}=0$. The peak is suppressed if $T$ or $B_{\|}$are increased. $g(T)$ and $g\left(B_{\|}\right)$at $V_{\text {bias }}=0$ are plotted in figures $6(\mathrm{c})$ and (d). A zero-bias peak and its suppression by $T$ and $B_{\|}$is a hallmark of Kondo phenomena. Landé factor $g^{*} \approx 0.3$ in the point contact is too small to result in a detectable Zeeman splitting of the zero-bias anomaly in our samples.

\subsection{Static polarization of '0.7 structure'}

Experimentally, it is possible to clarify the origin of ' 0.7 structure' by measuring polarization of carriers emerging from the QPC using magnetic focusing technique discussed above. Dependence of the first focusing peak on the injector conductance is shown in figure 7(b). The top curve is measured with conductance of both QPC1 and QPC2 being tuned into the first quantized plateau $G=g_{0}$. Both peaks have approximately the same value, consistent with the expectation that at $G=g_{0}$
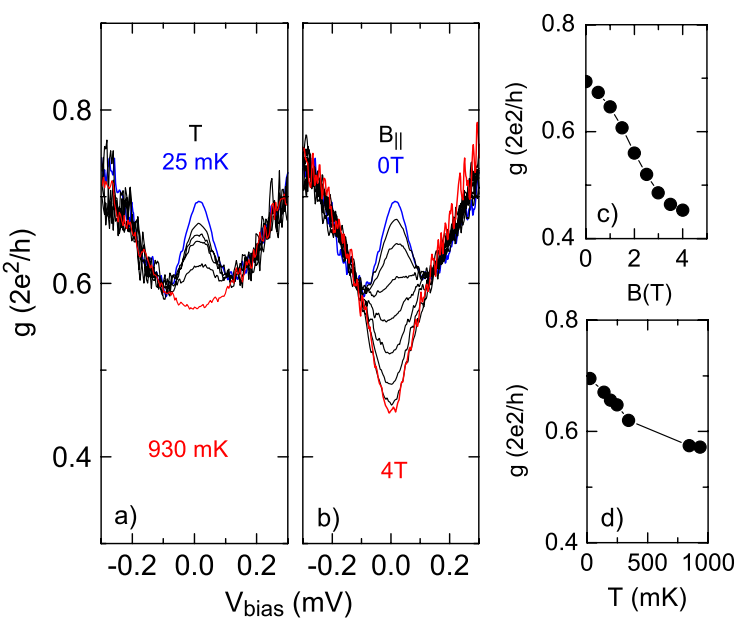

Figure 6. Differential conductance $g=\mathrm{d} I / \mathrm{d} V$ is measured as a function of dc bias $V_{\text {bias }}$ across the QPC1. Gate voltage $V_{\mathrm{g} 1}$ is fixed in the middle of the $0.7 \times 2 e^{2} / h$ plateau at $T=25 \mathrm{mK}$ and $B_{\|}=V_{\text {bias }}=0$. In (a) $B_{\|}=0$ and $T=25,140,190,250,340$ and $930 \mathrm{mK}$, in (b) $T=25 \mathrm{mK}$ and $B_{\|}$changes between 0 and $4 \mathrm{~T}$ in steps of $0.5 \mathrm{~T}$. Zero-bias anomaly is the strongest at the lowest $T$ and $B_{\|}=0$ and is suppressed as $T$ and/or $B_{\|}$increases. (c) and (d) $B$ and $T$ dependence of $g$ at $V_{\text {bias }}=0$.
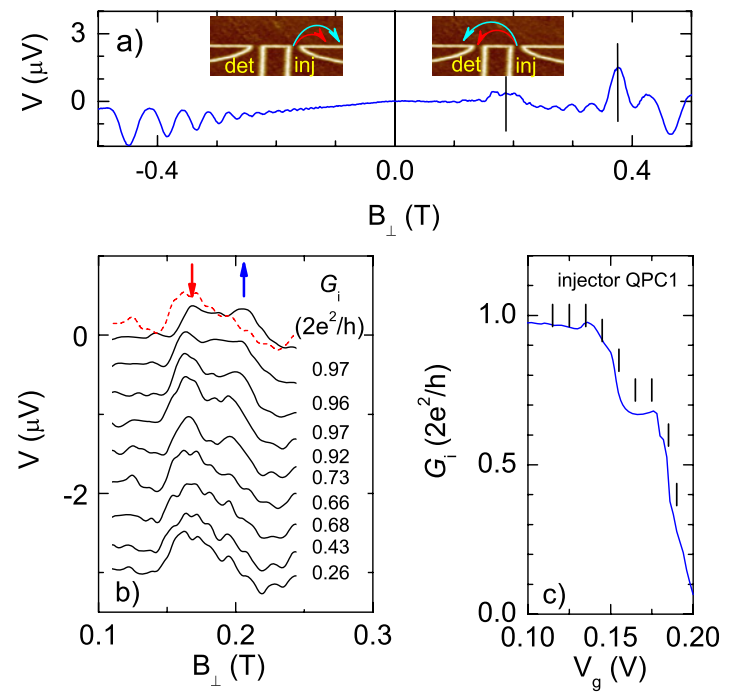

Figure 7. Polarization detection via magnetic focusing. (a) Voltage across the detector QPC2 is measured as a function of perpendicular magnetic field $\left(B_{\perp}\right)$. Current of $0.5 \mathrm{nA}$ is flowing through the injector QPC1. Positions of the first two magnetic focusing peaks are marked with vertical lines. Trajectories of the ballistic holes for positive and negative $B_{\perp}$ are shown schematically in the insets. (b) The first focusing peak is measured at different injector conductances with the detector tuned into the middle of the $2 e^{2} / h$ plateau. The curves are vertically offset by $-0.4 \mu \mathrm{V}$ relative to the top one. The $G=0.66 g_{0}$ curve is also plotted without an offset (red dashed line). (c) Gate voltage characteristic of QPC1, vertical lines mark positions where curves in (b) are taken.

there are two fully transmitting spin states below the Fermi energy. We fix the detector QPC2 at $G_{\mathrm{d}}=g_{0}$ to allow both spin states to be detected and gradually reduce conductance of the injector QPC1 to $G_{i}<g_{0}$. As $G$ decreases, height of 


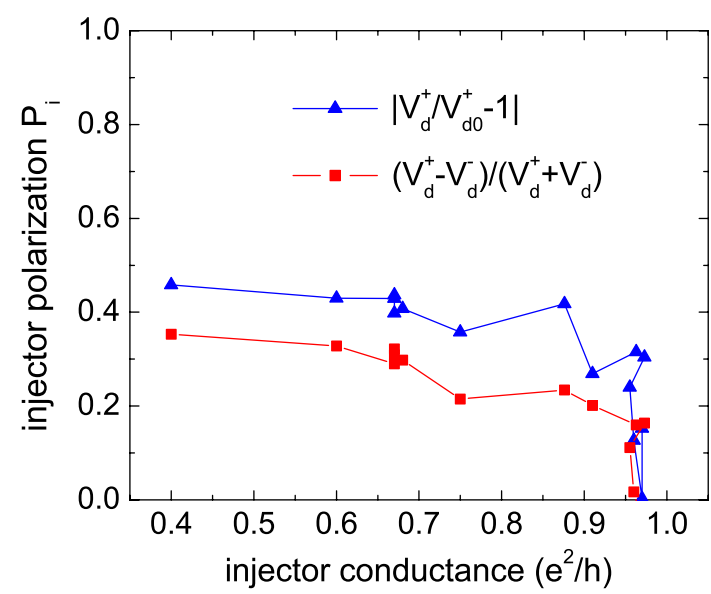

Figure 8. Polarization of the injector QPC1 as a function of conductance $G_{i}$ determined using equations (4) and (5).

the high- $B$ peak within the first focusing peak decreases, while height of the low- $B$ peak increases. This indicates that the two subbands with opposite spins are not equally populated at $G<g_{0}$ and, thus, there is a finite polarization of holes injected from QPC1. Polarization of the injector QPC is extracted using equations (4) and (5) and is plotted in figure 8. Note that polarization due to Zeeman splitting of spin subbands in an external magnetic field is too small to be detected in our experiments, $g^{*} \mu_{\mathrm{B}} B_{\perp} \approx 6 \mu \mathrm{eV} \lesssim k_{\mathrm{B}} T, e V_{\mathrm{ac}}$. Also, we do not expect hyperfine interaction to play significant role since the leading contact Fermi term is absent for holes.

We conducted several tests to insure that the extracted polarization is not dominated by disorder-mediated fluctuations. The reported data was reproducible over several thermal cyclings to room temperature (six for the sample in figure 7). Switching injector and detector with simultaneous reversal of magnetic field results in almost identical magnetic focusing data. $P_{\mathrm{i}}^{(2)}$, calculated from each peak at the same $B$ using equation (5) is consistent with the $P_{\mathrm{i}}^{(1)}$ calculated from both peaks $\Delta B=30 \mathrm{mT}$ apart but at the same gate voltage, equation (4), see figure 8 . While changes in the field and gate voltage are comparable with the period of mesoscopic fluctuations in similar structures, the agreement between the two measurements indicates that the observed polarization of $30-40 \%$ for $G_{i}<g_{0}$ is real and is not a result of spurious mesoscopic effects. Asymmetric gating of the point contact shifts the conducting channel in space and, thus, allows us to scan through the underlying disorder potential [35]. Changing $V_{\mathrm{gc}}-V_{\mathrm{g} 1}$ by $90 \mathrm{mV}$ shifts the channel by $\approx 7 \mathrm{~nm}$, while the correlation length for the disorder inside a $1 \mathrm{D}$ channel in a similar but higher mobility electron samples was measured $\approx 2 \mathrm{~nm}$. In our sample this shift also translates into an extra half-flux quanta being inserted inside the focusing trajectory. Experimentally, the peak heights remain the same as we laterally shift the injector channel (although peaks become slightly broader), see figure 4. Finally, peak height is sensitive to the in-plane magnetic field (see inset in figure 3 ), which is expected for spin subbands but not for mesoscopic fluctuations. Thus, our experiments provide a direct measurement of finite polarization in point contacts.
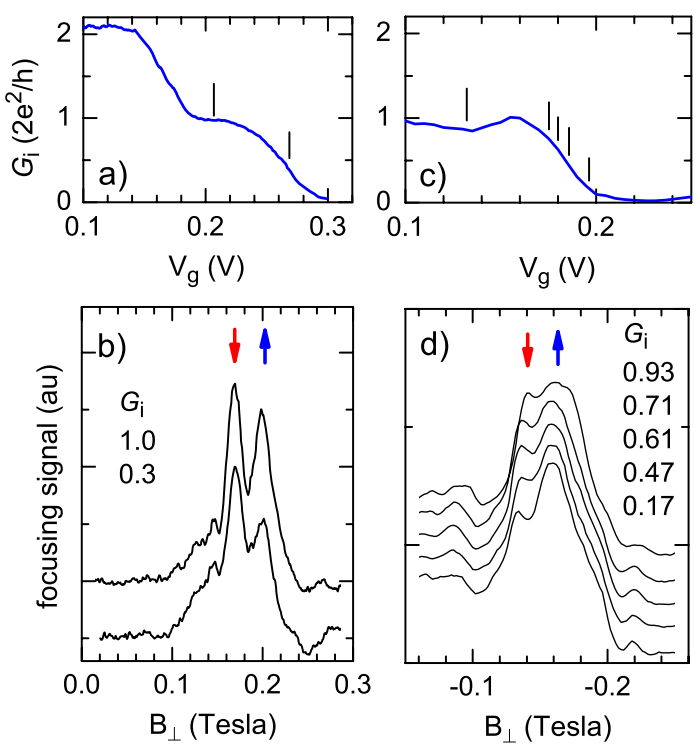

Figure 9. Polarization in samples with no well defined ' 0.7 structure'. (a) and (c) Conductance of injector QPCs for two samples. (b) and (d) The first focusing peak is plotted for fixed $G_{\mathrm{d}}=2 e^{2} / h$ and $G_{i}$ as indicated in the labels (in units of $2 e^{2} / h$ ). Vertical lines in (a) and (c) mark positions where the corresponding curves in (b) and (d) are taken. Curves in (b) and (d) are offset for clarity.

\subsection{Finite polarization in the absence of ' 0.7 structure'}

Appearance of a plateau around $0.7 g_{0}$ requires substantial energy splitting between the two spin subbands, comparable to or larger than the level broadening. In many QPCs, though, this condition is not met and there is no extra plateau below $g_{0}$. The question remains whether there is still a finite polarization below the first quantized plateau. We investigated several QPCs with no '0.7 structure', see figure 9. Samples are fabricated from different wafers A (left panel) and B (right panel). Injector QPCs in both devices have well defined first quantized plateau at $2 e^{2} / h$ but no '0.7 structure'. Magnetic focusing signal is measured with the detector QPC fixed at $G_{\mathrm{d}}=g_{0}$. At $G_{i}=g_{0}$ the first focusing peak is split in two peaks of similar height, with both spin subbands being populated. As $G_{i}$ is decreased below $g_{0}$ one of the peaks becomes suppressed while the other enhances, similar to the device with well defined ' 0.7 structure'. Polarization $P_{\mathrm{i}}$ increases gradually from 0 to $\sim 15 \%$ as $G_{i}$ decreases from $1 g_{0}$ to $0.2 g_{0}$; polarization for this sample is plotted in figure 10 and is approximately twice lower than in the device with '0.7 structure'. We conclude that polarization of QPCs near the onset of the conduction is a rather generic property and appearance of the ' 0.7 structure' is an extreme indicator of such polarization when spin gap becomes large enough to result in a measurable feature in the gate voltage characteristic.

The two devices in figure 9 have different crystallographic orientations and, thus, different angles between the momentum of the injected carriers and the internal SO field $\left(I_{i} \|[\overline{2} 33]\right.$ and $I_{i} \|[0 \overline{1} 1]$ for the samples on the left and right panels) which, presumably, results in a different peak being suppressed. For the device to work as a spin detector it is sufficient that each spin state in a QPC adiabatically maps on one of the chiral 


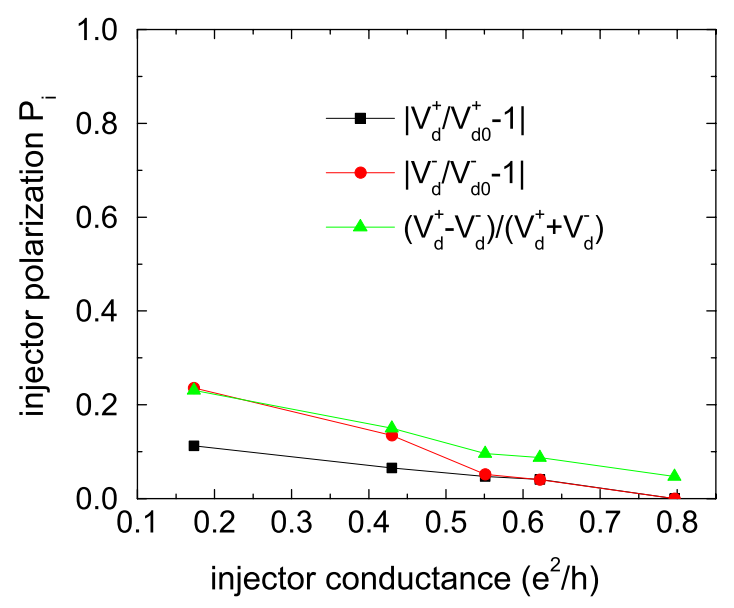

Figure 10. Polarization of the injector in the sample from figure 9 as a function of conductance $G_{i}$ determined using equations (4) and (5).

states in the adjacent 2D gas, which has been checked by application of a strong Zeeman field as discussed in [27]. The exact mapping conditions are the subject of ongoing research.

\section{Conclusions}

In conclusion, we present an experimental investigation of '0.7 structure' in p-type QPCs with a new twist: a direct measurement of spin polarization. Using a newly developed spin separation technique we determine the polarization of holes injected from a QPC into an adjacent 2D gas. The technique is sensitive to static polarization, which is found to be as high as $40 \%$ in samples with well defined ' 0.7 structure'; some polarization has been measured in all point contacts below the first plateau. This result questions the Kondo interpretation as an origin of ' 0.7 structure', which is incompatible with a finite static polarization. The ' 0.7 structure' in p-type QPCs shows all the essential features reported for n-type QPCs, such as gradual evolution into $0.5 g_{0}$ plateau at high in-plane magnetic fields, survival at high temperatures, gradual increase toward $1.0 g_{0}$ at low temperatures, and zero-bias anomaly, which is suppressed by either temperature increase or application of a magnetic field. The similarities between p-type and n-type QPCs suggest that the underlying physics responsible for the appearance of ' 0.7 structure' should be the same.

\section{Acknowledgments}

The authors thank Yu Lyanda-Geller for valuable discussions. This work was supported by NSF grant ECS-0348289.

\section{References}

[1] Weinmann D, Häusler W and Kramer B 1995 Spin blockades in linear and nonlinear transport through quantum dots Phys. Rev. Lett. 74 984-7

[2] Rokhinson L P, Guo L J, Chou S Y and Tsui D C 2001 Spin transitions in a small Si quantum dot Phys. Rev. B $\mathbf{6 3} 035321$
[3] Huttel A K, Qin H, Holleitner A W, Blick R H, Neumaier K, Weinmann D, Eberl K and Kotthaus J P 2003 Spin blockade in ground-state resonance of a quantum dot Europhys. Lett. $62712-8$

[4] Papadakis S J, de Poortere E P, Manoharan H C, Shayegan M and Winkler R 1999 The effect of spin splitting on the metallic behavior of a two-dimensional system Science $2832056-8$

[5] Thomas K J, Nicholls J T, Simmons M Y, Pepper M, Mace D R and Ritchie D A 1996 Possible spin polarization in a one-dimensional electron gas Phys. Rev. Lett. 77 135-8

[6] Kristensen A, Bruus H, Hansen A F, Jensen J B, Lindelof P E, Marckmann C J, Nygard J, Sorensen C B, Beuscher F, Forchel A and Michel M 2000 Bias and temperature dependence of the 0.7 conductance anomaly in quantum point contacts Phys. Rev. B 62 10950-7

[7] Cronenwett S M, Lynch H J, Goldhaber-Gordon D, Kouwenhoven L P, Marcus C M, Hirose K, Wingreen N S and Umansky V 2002 Low-temperature fate of the 0.7 structure in a point contact: a Kondo-like correlated state in an open system Phys. Rev. Lett. 88226805

[8] Roche P, Segala J, Glattli D C, Nicholls J T, Pepper M, Graham A C, Thomas K J, Simmons M Y and Ritchie D A 2004 Fano factor reduction on the 0.7 conductance structure of a ballistic one-dimensional wire Phys. Rev. Lett. 93116602

[9] van Wees B J, van Houten H, Beenakker C W J, Williamson J G, Kouwenhoven L P, van der Marel D and Foxon C T 1988 Quantized conductance of point contacts in a two-dimensional electron gas Phys. Rev. Lett. 60848

[10] Wharam D A, Thornton T J, Newbury R, Pepper M, Ahmed H, Frost J E F, Hasko D G, Peacock D C,

Ritchie D A and Jones G A C 1988 One-dimensional transport and the quantisation of the ballistic resistance J. Phys. C: Solid State Phys. 21 L209-14

[11] Chou H T, Luscher S, Goldhaber-Gordon D, Manfra M J, Sergent A M, West K W and Molnar R J 2005 High-quality quantum point contacts in $\mathrm{GaN} / \mathrm{AlGaN}$ heterostructures Appl. Phys. Lett. 8673108

[12] Reilly D J, Buehler T M, O'Brien J L, Hamilton A R, Dzurak A S, Clark R G, Kane B E, Pfeiffer L N and West K W 2002 Density-dependent spin polarization in ultra-low-disorder quantum wires Phys. Rev. Lett. 89246801

[13] Lieb E and Mattis D 1962 Theory of ferromagnetism and the ordering of electronic energy levels Phys. Rev. 125 164-72

[14] Spivak B and Zhou F 2000 Ferromagnetic correlations in quasi-one-dimensional conducting channels Phys. Rev. B 61 16730-5

[15] Berggren K F and Yakimenko I I 2002 Effects of exchange and electron correlation on conductance and nanomagnetism in ballistic semiconductor quantum point contacts Phys. Rev. B 6685323

[16] Klironomos A D, Meyer J S and Matveev K A 2006 Spontaneous spin polarization in quantum wires Europhys. Lett. 74 679-85

[17] Meir Y, Hirose K and Wingreen N S 2002 Kondo model for the ' 0.7 anomaly' in transport through a quantum point contact Phys. Rev. Lett. 89196802

[18] Rejec T and Meir Y 2006 Magnetic impurity formation in quantum point contacts Nature 442 900-3

[19] Yoon Y, Mourokh L, Morimoto T, Aoki N, Ochiai Y, Reno J L and Bird J P 2007 Probing the microscopic structure of bound states in quantum point contacts Phys. Rev. Lett. 99136805

[20] Seelig G and Matveev K A 2003 Electron-phonon scattering in quantum point contacts Phys. Rev. Lett. 90176804

[21] Matveev K A 2004 Conductance of a quantum wire in the Wigner-crystal regime Phys. Rev. Lett. 92106801 
[22] Sharvin Yu V 1965 A possible method for studying Fermi surfaces Zh. Eksp. Teor. Fiz. 48984

Sharvin Yu V 1965 Sov. Phys._JETP 21655

[23] Tsoi V S 1975 Determination of the dimensions of nonextremal Fermi-surface sections by transverse focusing of electrons JETP Lett. 22197

[24] Tsoi V S, Bass J and Wyder P 1999 Studying conduction-electron/interface interactions using transverse electron focusing Rev. Mod. Phys. 71 1641-93

[25] Van Houten H, Beenakker C W J, Williamson J G, Broekaart M E I, Van Loosdrecht P H M, Van Wees B J, Mooij J E, Foxon C T and Harris J J 1989 Coherent electron focusing with quantum point contacts in a $2 \mathrm{~d}$ electron gas Phys. Rev. B 398556

[26] Goldman V J, Su B and Jain J K 1994 Detection of composite fermions by magnetic focusing Phys. Rev. Lett. 722065

[27] Rokhinson L P, Larkina V, Lyanda-Geller Y B, Pfeiffer L N and West K W 2004 Spin separation in cyclotron motion Phys. Rev. Lett. 93146601

[28] Altshuler B L, Aronov A G, Larkin A I and Khmelnitskii D E 1981 Anomalous magnetoresistance in semiconductors $Z h$. Eksp. Teor. Fiz. 81768

Altshuler B L, Aronov A G, Larkin A I and Khmelnitskii D E 1981 Sov. Phys._JETP 54411
[29] Bychkov Yu A and Rashba E I 1984 Properties of a 2d electron gas with lifted spectral degeneracy Pis. Zh. Eksp. Teor. Fiz. 3966

Bychkov Yu A and Rashba E I 1984 JETP Lett. 39 78-81

[30] Zülicke U, Bolte J and Winkler R 2007 Magnetic focusing of charge carriers from spin-split bands: semiclassics of a Zitterbewegung effect New J. Phys. 9355

[31] Potok R M, Folk J A, Marcus C M and Umansky V 2002 Detecting spin-polarized currents in ballistic nanostructures Phys. Rev. Lett. 89266602

[32] Snow E S and Campbell P M 1994 Fabrication of Si nanostructures with an atomic force microscope Appl. Phys. Lett. 64 1932-4

[33] Held R, Heinzel T, Studerus A P, Ensslin K and Holland M 1997 Semiconductor quantum point contact fabricated by lithography with an atomic force microscope Appl. Phys. Lett. 71 2689-91

[34] Rokhinson L P, Tsui D C, Pfeiffer L N and West K W 2002 AFM local oxidation nanopatterning of a high mobility shallow 2d hole gas Superlatt. Microstruct. 32 99-102

[35] Heinzel T, Salis G, Held R, Luscher S, Ensslin K, Wegscheider W and Bichler M 2000 Shifting a quantum wire through a disordered crystal: observation of conductance fluctuations in real space Phys. Rev. B 61 R13353-6 